\title{
Microscopic Traffic Behaviour near Incidents
}

\author{
Victor L. Knoop, Henk J. van Zuylen and Serge P. Hoogendoorn, TRAIL \\ research school, Delft University of Technology, Delft, The Netherlands
}

\begin{abstract}
Much of the delays on road networks are caused by incidents. This is partially caused by blockage or closure of lanes, but also by the change of driving behaviour in the remaining lanes. This contribution analyses traffic flow conditions near an incident both microscopically and macroscopically. A theory is proposed to describe drivers' behaviour, which is tested using traffic data of individual vehicles, collected using a helicopter. A bimodal headway distribution is observed, centred around two mean values, 2 seconds and 4 seconds. To understand the underlying mechanisms a car-following model is fitted to the drivers' behaviour. The model parameters show that the reaction time is much higher than usual. Using this model-based analysis, we conclude that the incident distracts the drivers and less attention is paid to the driving process. The consequence is that the queue discharge rate for the unblocked lanes is $30 \%$ lower than the usual queue discharge rate per lane.
\end{abstract}

\section{Introduction}

A significant part of road-users' delays is caused by incidents, see for instance Kwon et al. (2006). Incidents cause delays because the road capacity is reduced, caused by physically blocked lanes, but also caused by a different driving behaviour around incidents. To get a good understanding of this phenomenon, empirical research would be most suitable.

The Highway Capacity Manual (2000) provides estimates for capacities around incidents. Qin and Smith (2001) carry out a more detailed analysis, but it still is a macroscopic analysis based on macroscopic data. Incidents are also modelled in micro-simulation packages. Using several of these software tools, Sinha et al. (2007) find a capacity reduction of about $50 \%$ if one of three lanes is blocked. The empirical reduction found by Knoop et al. (2008) is around $65 \%$.

Given the observed changes in macroscopic flow characteristics, we know that drivers change their behaviour when driving along an incident site. However, to gain more insight into these behavioural changes, empirical microscopic traffic data is required. To the best of our knowledge, microscopic measurements of the 
behaviour of single vehicles around incidents have not been collected and analysed in detail until now. The change in individual driving behaviour causes a change in the macroscopic traffic flow characteristics of the road, such as the capacity. It is still a scientific challenge to describe traffic flow along incidents, especially at the level of individual drivers. This contribution takes on this challenge. For the first time, a theory is proposed for microscopic driving behaviour around incidents which is tested with real-life data. This conceptual model is proposed and then tested using data of individual vehicles in real life.

To this end, we have collected empirical trajectory data using a digital camera mounted under a helicopter. At two incident locations, the traffic operations have been captured on high resolution video from the helicopter which flew high enough not influence the traffic operations. From the video, we derived trajectories, as described by Hoogendoorn et al. (2003).

We have calibrated a car-following model for these incident locations. At first, data is analysed at the level of individual vehicles and drivers. We also show considerable differences in individual driving behaviour between driving under normal conditions and driving along an incident site. We show how these changes translate into changes in macroscopic properties which are also found in the data.

The behaviour of the traffic on the carriageway in both directions has been analysed. Particularly the behaviour on the carriageway which is not blocked is of interest from a behavioural point of view. Since there is no physical obstruction that causes a change in traffic operations, the changes are solely due to a changed driving behaviour. Analysing the effects on this carriageway quantifies the effects of "rubbernecking".

Although drivers might act differently at another incident site, the change in driving found in these two cases appears to have generic features and may have a certain generic validity for application to a different location. The qualitative changes are likely to be the same, although quantitatively, effects may vary (for instance on the incident type, the presence of emergency services etc.). More importantly, it shows the microscopic mechanisms that cause the flow to change and thus which parameters to tune to calibrate a model for incident situations.

The remainder of the paper is set-up as follows. Section 2 poses the hypotheses that are tested in the contribution, section 3 describes the experimental setup. Section 4 and 5 are closely related and describe respectively the data processing and the results. If the reader prefers, these sections can be read together, paragraph by paragraph. Section 6 summarizes the paper and gives the concluding remarks.

\section{Theory and behavioural hypotheses}

This contribution proposes a theory predicting how driving behaviour around incidents is likely to differ from normal conditions. In our theory, drivers shift their attention towards the incident when passing by the incident site. The degree of at- 
tention shift differs per driver, with some drivers only focussing on the traffic whereas others focus mainly on the incident.

This attention shift changes the driving behaviour at incident sites in a way depicted in Fig. 1. We propose that the drivers are distracted and due to the limited attentional resources, the attention for the driving task reduces. Two changes now take place. Firstly, the reaction time increases. Secondly, the risk of an accident which involves themselves increases. The drivers therefore choose to have a larger headway. In practice, drivers could obtain a larger headway by decreasing speed, in which case an equal space headway becomes a larger time headway or alternatively by changing lanes. These driver characteristics change the macroscopic properties of the flow. An increased minimum headway causes a reduced capacity, a decreased speed causes a decreased average speed and a lane change will change the distribution of vehicles across the available lanes. In congested flow conditions all lanes are occupied and one cannot change towards a quiet lane. Since the measurements presented here are taken in congestion, the part of the model about lane changing will not be discussed here

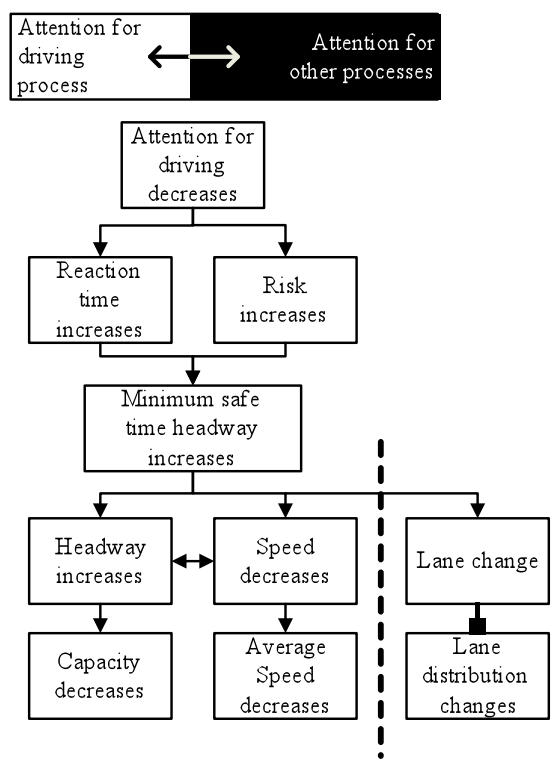

Fig. 1 Mechanism relating attention level to macroscopic flow characteristics.

The theory and the other parts of the model are tested using 4 hypotheses about the driving behaviour stated below:

1. The distribution of headways in a bottleneck caused by the incident is different compared to a bottleneck in normal traffic and the mean headway is larger.

2. The reaction time distribution of drivers is different compared to normal traffic and the mean is larger. 
3. The sight of an incident site reduces average speed.

4. The queue discharge rate at the incident site is lower than the outflow capacity under normal conditions for the same roadway geometry.

Hypotheses 1 and 2 are based on individual driving behaviour, whereas hypotheses 3 and 4 are macroscopic properties. The summary of the data that have been extracted is given below in Table 1 where for each of the hypotheses the used indicator is stated. The indicators have been compared with the same indicators for normal traffic. For one indicator at one site, we had to use a literature value for the reference situation stating an average value for the Netherlands as well as the spread. For all other indicators, a comparable indicator is found, although they are sometimes derived from data at another location, but always with a comparable driver population. This was needed since the traffic conditions at the incident sites differ from the conditions at the same locations in non-incident situations, and therefore, at same sites no comparable data for non-incident situations could be derived. The reference situations now are chosen such that there are the same traffic conditions as in the incident situations.

Table 1. The used indicators

\begin{tabular}{ll}
\hline Hypothesis & Indicator \\
\hline 1. Headways & Headway distribution \\
2. Reaction time distribution & Results of a fit of a car-following model \\
3. Traffic flow speed & Average speed profile and interval bounds \\
4. Outflow capacity & Outflow capacities and spread \\
\hline
\end{tabular}

\section{Experimental Set-Up}

This section discusses the required measurements for this study. The first part discusses the necessity of detailed trajectory information in order to gain insight into the macroscopic and microscopic traffic flow operations near incidents. The second part introduces the observations that are used for this paper and in the third part the properties of the resulting data are given.

\subsection{Need for Trajectory Data}

There are many ways to measure traffic behaviour. Recently, Schaap et al. (2008) showed how drivers react on an unexpected event in a driving simulator. They showed how an unexpected action of a leading vehicle influences the driving over longer time. We prefer to measure the effects of incident in real-world. Loop de- 
tectors are the most common way to gather traffic data, but these will not provide sufficiently detailed information about the driving behaviour dynamics around incidents, since they only provide local, cross-sectional, information. Spatial information, or, in fact, trajectory information, is needed, because this will yield information of the behaviour upstream of the incident and at the incident location itself. This allows for instance to observe speed adaptation and car-following behaviour, including estimation of the reaction times.

For microscopic behaviour, it is essential to follow a single vehicle and its leaders over a certain time period. Only from the exact place of the vehicle and the leaders, one can derive the stimuli that are possibly input for the driver to accelerate. To derive the acceleration, one needs a time series of exact position measurements.

For the capacity estimation passing times are sufficient. However, data from one loop detector will not be sufficient, since one needs to know whether there is a queue waiting; this information cannot be derived from one local measurement.

\subsection{Description of Incidents}

The following approach was taken to get the trajectory data of an incident (Hoogendoorn et al., 2003). A digital photo camera was attached to a helicopter. The helicopter stayed approximately at the same position, above the incident. The camera could move in all directions to compensate for the helicopter movements. Digital photographs were taken at a rate of 15.1 images per second and saved to a hard drive. The size of the pictures is 1392 x 1040 pixels. The height of the helicopter is around $400 \mathrm{~m}$ and length of the long size of the image is also around 400 meters. This implies that one pixel equals around $30 \times 30 \mathrm{~cm}$ on the road.

The observation team waited at the Traffic Management Centre in the centre of the Netherlands until an incident was reported nearby, after which it flew with the helicopter to the incident location. From the moment of arrival, traffic operations for both directions have been recorded. For traffic in the non-incident direction, the incident was visible but it formed no physical obstruction. The video shows the so-called "rubbernecking effect" (i.e. people watching the incident at the other side of the guardrail). The altitude of the helicopter was large enough not influence the traffic operations.

The remainder of the paper presents the data for two incidents. All properties are summarized in Table 2 below. At the first incident a van rolled over at 6 June 2007, at around 9:15 am, near the city of Apeldoorn at the motorway A1 in the Netherlands. It ended in the median strip, the unpaved area between the two carriageways of the motorway. The road has two carriageways in each direction and no gradient. Congestion occurred in both directions with the heads of both queues at the location of the incident which means that the incident formed an bottleneck. For the eastbound direction, the emergency vehicles blocked one lane which was 
therefore unavailable for the traffic. For the other, westbound, direction, the delay was only caused by rubbernecking. Since there was a tunnel around 100 meters west of the blocking of the lane, the traffic operations there were invisible (see Fig. 2). The crosses mark the blocked lanes, the arrows the available lanes.

Table 2. Properties of the incidents for which data are collected.

\begin{tabular}{lll}
\hline Nearby city & Apeldoorn & Gorinchem \\
\hline Road & A1 & A15 \\
Date & 6 June 2007 & 6 June 2007 \\
Time & $9: 39-10: 15$ & $11: 59-12: 56$ \\
Weather & Clear & Clear \\
Type & Motorway & Motorway \\
Number of lanes & $2 \times(2+$ shoulder $)$ & $2 \times(2+$ shoulder $)$ \\
Gradient & none & none \\
Weather & Clear & Clear \\
Lanes used by traffic: & & \\
\multicolumn{1}{c}{ eastbound } & right \& shoulder & right \& left \\
westbound & left \& right & left \\
Jammed & eastbound \& westboundwestbound \\
imaged area & 400 m & 400 m \\
Remarks & just east of tunnel & just east of merging \\
\hline
\end{tabular}

The lanes used changed over time for traffic in the eastbound direction. Sometimes, the shoulder lane was used, sometimes, the right lane was used, and sometimes, they were used both. From the video we have observed that some drivers choose to avoid a specific lane without an apparent reason. Both lanes have been available for the drivers. Taking the flow values of each of the lanes separately would yield nonsensical results (since often no-one uses the lane, although there is a queue waiting).

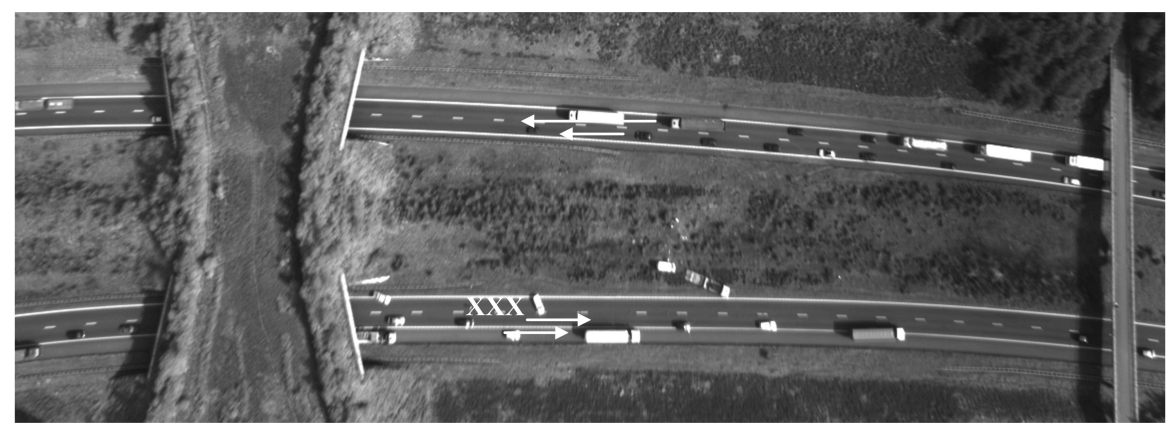

Fig. 2The incident location near Apeldoorn (right=east). 
The second incident happened at 6 June 2007, at around $11 \mathrm{am}$, at the westbound carriageway of the two-lane motorway A15. In this incident, several trucks and passenger cars were involved which blocked one lane of the two-lane motorway as well as the shoulder lane. From time to time, the police stopped the traffic for a while to recover a car from the incident. It happened several hundreds of meters downstream of the freeway junction Gorinchem (see Fig. 3); there is no gradient. Traffic had to merge twice in several hundreds of meters: the regular merging of traffic from the two freeways and the merging from two to one lane. The traffic demand in the eastbound direction was not sufficiently high to cause a traffic jam.

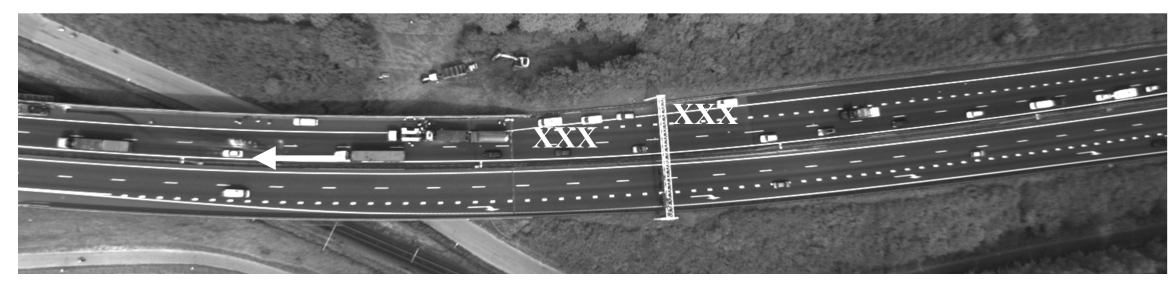

Fig. 3 Incident location near Gorinchem (right=east)

\subsection{Description of the Data}

The video was taken from a helicopter. This means that the image area is not stable. Due to this movement of the recorded area we could not use the full length of the recorded road image. Around 200-300 meters of the road remains that overlapped all recorded image and the full length of the available image is used to track the vehicles. This means that typically, a vehicle appears on 200 different images before it reaches the end of the analysed stretch.

Table 3. The number of vehicles used for computing average speeds and the number of leaderfollower pairs used for determining headways and calibrating parameters of a car-following model.

\begin{tabular}{|c|c|c|c|c|}
\hline & Eastbd. right lane & Eastbd. left lane & Westbd. right lane & Westbd. left lane \\
\hline Apeldoorn & 644 (headways & s, left + right) & $\begin{array}{l}76 \text { (car-following) } \\
366 \text { (headways) }\end{array}$ & $\begin{array}{l}123 \text { (car-following) } \\
331 \text { (headways) }\end{array}$ \\
\hline Gorinchem & 378 (speed) & 90 (speed) & - & $\begin{array}{l}123 \text { (car-following) } \\
402 \text { (headways) }\end{array}$ \\
\hline
\end{tabular}

The operations of several hundreds of vehicles were recorded on video. Table 3 states how many vehicles were used in the further analysis. It also shows there are differences in the number of vehicles used for further analysis depending on the type of analysis. For car-following analysis, for instance, one needs the complete 
trajectories of a leader-follower pair, whereas for the headways one only needs to know the headway of a leader-following pair at one moment in time.

Due to the resolution of the images, the trajectories have to be smoothed before they can be analysed (Thiemann et al., 2008). To this end, the same filtering is applied as described by Ossen et al. (2006) and Toledo et al. (2007) where the position of the vehicle at a certain moment in time was replaced by a weighted average of the position of the vehicle at neighbouring times. The weighting decreased for times further away, with a typical decrease of 1 second, which means that when determining the position at time $t$, the positions of the vehicle at $t+1$ second and $t-$ second get half the weight of the vehicle's position at moment $t$. The positions at times which differ more than 2 seconds from time $t$ are not considered at all.

\section{Data Analysis}

Both the behaviour of the drivers (microscopic) and the characteristics of the flow (macroscopic) have been studied. At the microscopic level, the chosen time headway at one location and the dynamic car-following characteristics are studied, which is described in section 4.1. Section 4.2 discusses the way the macroscopic properties of traffic are processed. The results are stated in section 5 which ordered the same way as this section, which therefore can be read together.

\subsection{Microscopic Flow Characteristics}

The basis for all changes in traffic flow is the change of human behaviour when driving. Differences in capacities can be derived from differences in headways of individual drivers, which are likely to be caused by differences in dynamical carfollowing behaviour. This section presents how insights at this microscopic level can be gained. It is divided in three subsections, of which the first discusses the headways, of which the results can be found in section 5.1.1. Section 4.1.2 discusses how a car-following model can be calibrated. The third subsection finally discusses the robustness of the parameter values found in the calibrating process. Results of the calibrating process are given in section 5.1.2.

\subsubsection{Headways}

The trajectory data of the vehicles contains, by interpolation, the moment a vehicle passes a predetermined point, which will be called a "virtual detector". For all vehicles for which the trajectories are reconstructed, passing times are collected. In case a leader-follower combination is tracked, the difference of the passing times 
times gives the headway. This headway could be analysed at any point along the trajectory. The most interesting point was chosen, which is the point that forms the bottleneck, found by the moment that cars start accelerating again. Note that this point is a fixed point and does not vary over time.

We have analysed the distribution of headways (in the congested directions) in order to check whether the headways around an incident are larger compared to normal driving. Apart from the average value, the distribution of the headways is interesting. One possibility is that every driver takes a longer headway, whereas another is that some people keep normal headways and others keep very long headways.

The headway distribution only is relevant if a bottleneck is present in the monitored area and people are bound to a lane. This is the case for the following three lanes:

- Apeldoorn westbound right lane

- Apeldoorn westbound left lane

- Gorinchem westbound left lane

It is only relevant to analyse headways collected at a bottleneck itself. For instance upstream of a bottleneck every vehicle could be in car-following mode, since there is congestion, but nevertheless the average headway is determined downstream at the bottleneck. We did not have access to remote sensing data of individual drivers' behaviour at a comparable bottleneck or, in fact, at any bottleneck. However, the median headway for traffic flowing out of a queue is the inverse of the median queue discharge rate, which is the maximum number of vehicles per unit time flowing out of the queue.

The queue discharge rate can be determined from loop detector data if both free flow and congested conditions occur. The road at both incident sites is equipped with loop detectors. At the Gorinchem site, there is sometimes congestion from downstream which spills back In contrast, at the Apeldoorn site there is no congestion at all. We therefore have to use a value from study to the queue discharge rate and the spread thereof on Dutch motorways. There is no reason why the queue discharge rate at the Apeldoorn site would be different from other Dutch motorways.

At the Apeldoorn site in the eastbound direction, the lane discipline is insufficient to get a good headway analysis. People drive at the right lane, at the shoulder lane or sometimes even between these lanes. Directly after they have passed the incident location, they change lanes. Therefore, a leader-follower couple could not be identified for a long time and the follower is likely to keep adjusting his headway to changing leaders. An estimate of the car-following model on this fluctuating behaviour would give unreliable outcomes, so we have not used data of Apeldoorn eastbound. For the Gorinchem eastbound direction there was not enough traffic to make a car-following analysis useful. Only a small fraction of the travellers was in car-following mode. 


\subsubsection{Calibrating a car-following model}

The longitudinal driving behaviour at the location of the bottleneck determines the headway distribution and capacity, given that there are no lane changes. This driving behaviour can be described by a car-following model. To quantify the driving processes, a car-following model is fitted to the vehicles passing an incident location. To find the differences with normal driving, the parameters describing the operational driving are compared with the same parameters for driving under similar conditions without an incident. This section will explain the choice of the model and the fitting process.

The goal of fitting a model is that the fitted model parameters can be compared to the fitted parameters in normal, non-incident conditions. However, it is impossible to monitor the same drivers at the same site under the same conditions without an incident. The best option is to compare the parameters with parameters from a similar drivers' population at a similar site under similar driving conditions. However, it is impossible to collect comparable data from the incident sites since they are usually uncongested. Therefore, we compared the model parameters with parameters obtained from an observation at other, but comparable sites in terms of road geometry, driving conditions and drivers' population, which is the Everdingen and Waalhaven site as presented in Ossen (2008).

For describing the longitudinal driving behaviour around the incident location, a model with both a car-following part and a free driving part is required, since upstream of the incident traffic is congested and downstream of the incident it is not congested. Furthermore, it is expected that the reaction time of drivers plays an important role. All these aspects are included in the Tampère (2004) carfollowing model.

In the Tampère model, shown in equation (1), the driver has a desired distance $s^{*}(v)$, which is linearly dependent on its own speed $v$. The time derivative of the speed, the lateral acceleration $\mathbb{E}$, is the minimum of two terms. One of these terms is a car-following term in which the desired acceleration is in fact a linear combination of two stimuli. One stimulus is the speed difference with the predecessor, $\Delta v$. The other stimulus in the same term is the difference between the actual distance with the predecessor, $\Delta x$, and the desired distance, $s^{*}(v)$. The other term is a free driving term. The acceleration is at maximum a constant times the difference between the desired or free speed, $v^{*}$, and the actual speed. A reaction time is taken into account by delaying the response to the stimuli by $\tau_{r}$. In mathematical terms, this model is expressed as follows:

$$
\begin{aligned}
s^{*}(v) & =c_{4}+c_{5} v \\
k\left(t+\tau_{r}\right) & =\min \left\{c_{1} \Delta v(t)+c_{2}\left(\Delta x(t)-s^{*}(v)\right), c_{3}\left(v^{*}-v(t)\right)\right\}
\end{aligned}
$$


We only need the model parameters to compare these with model parameters of the same model for driving under normal conditions. It is therefore not required that the Tampère model is a better model than another one. However, it is required that the model fits reasonably well on a vehicle. We therefore check on each vehicle whether the sensitivity of the fitting process for a parameter is high enough, which is explained in the next section. In case it is not, the vehicle is not considered in the further analysis.

This paragraph explains the way the fitting process is implemented, which is similar to the one described by Ossen et al. (2006). The goal of the optimization was to minimize an error function $\varepsilon$, which consisted of the error in predicted location and the error in the predicted speed. For each time step the next position and speed were calculated based on the measured trajectories of the follower and the leader up to that moment. If one would, in contrast, choose to predict the complete trajectory based on the trajectory of the leader and only the initial position and speed of the leader, an error at the beginning of the trajectory would accumulate and thus the errors at the beginning of the trajectory would get a larger weight. If the measured trajectory is used, like in our case, an error at the beginning does not accumulate and therefore is not more important than an error at the end of a trajectory.

The error is a function of trajectories of a leader-follower pair, which is fixed, a fixed reaction time and a set of 5 parameters. Using a standard algorithm in Mat$l a b$, fmincon, we find the minimum in this 5 dimensional space for each leaderfollower pair. The parameters have been bound to a feasible range which differed per variable, and a typical value has to be put in for the initial computation step.

This has been done for different reaction times in a certain range. For each reaction time an optimal parameters set $c_{1}-c_{5}$ has been found and corresponding remaining error. The reaction time with the minimum error has been selected as best and consequently the corresponding parameters $c_{1}-c_{5}$ as well as the corresponding reaction time are considered to be the best parameters to describe the follower's behaviour with the Tampère model.

\subsubsection{Robustness of the fitting parameters}

Even in the best fit, not all parameters of each vehicle could be estimated reliably. Therefore, formula (2) below shows how to compute the sensitivity $\mathrm{S}$ of the error for each of the 6 parameters. Note that in this equation, $\varepsilon^{n}$ is the error function for the nth vehicle and $c_{i}^{n}$ is any of the 6 parameters $c_{1}-c_{5}$ or the reaction time; this 6 parameters together are called $C^{n}$ and the optimal value of a parameter or a parameter set is indicated with a asterisk. The optimal set of parameters for the nth vehicle, $C^{n^{*}}$ is found if the derivative to each of the variables is 0 . How much the error fluctuates by a small change of the parameter is expressed in the second derivative. However, this shows the variation of the error when varying the parameter by one unit. Since the scales of the units differ per parameter, we can normalize this to a dimensionless parameter scale by multiplying the second derivative by 
the square of the value of the parameter. In order to obtain a scale without units, we then divide the sensitivity by the error value for the best parameter set, which gives the relative sensitivity of the parameters.

$$
S_{c_{i}}^{n}=\frac{c_{i}^{n^{*} 2}}{\varepsilon^{n}\left(C^{n^{*}}\right)} \frac{\partial^{2} \varepsilon^{n}}{\partial c_{i}^{2}}\left(C^{n^{*}}\right)
$$

The sensitivity shows how reliably a parameter can be estimated; in fact, a sensitivity is comparable with the inverse of a variance. We account for the sensitivity in the further analysis of the parameter values by introducing a weight factor $w$ which increases for increasing sensitivity, as proposed by Hoogendoon and Van Lint (2007). This weight is for instance used to compute a weighted average of a parameter over all vehicles. Additionally, parameters which could not be estimated reliably enough are neglected. The minimum sensitivity is 1 , a value for which the variation of the error is as large as the error when the parameter varies over one time the parameter value. These requirements are captured in the following weight function.

$$
w_{c_{i}}^{n}=\left\{\begin{array}{cc}
\log S_{c_{i}}^{n} & S_{c_{i}}^{n}>1 \\
0 & S_{c_{i}}^{n} \leq 1
\end{array}\right.
$$

For approximately $40 \%$ of the vehicles all 6 parameters can be calibrated reliably, i.e. for approximately $40 \%$ of the vehicles all 6 calibrated parameters have a sensitivity of larger than 1 . For the other vehicles it differs which parameters are sensitive; for 1 vehicle none of the parameters could not be calibrated reliably, for the others only some of the parameters could be calibrated. The calibration of $c_{4}$ and $c_{5}$ was the most reliable. $70 \%$ of all parameters could be estimated reliably.

We have chosen not to fit the desired speed. The sensitivity of the trajectory for the value of $v^{*}$, is low and therefore no reliable estimate for $v^{*}$ can be made; the only requirement for a plausible fit is that $v^{*}$ is not too low (Ossen, 2008). We therefore have fixed $v^{*}$ at a value near the speed limit, $30 \mathrm{~m} / \mathrm{s}$.

\subsection{Macroscopic Flow Characteristics}

This section discusses how changes in the average speed and the capacity can be found. The results can be found in sections 5.2.1 and 5.2.2 respectively.

\subsubsection{Average Speed}

A change in microscopic driving behaviour possibly shows a lower speed. For each point along the road, the average speed of the vehicles passing at that point has been computed. We have analysed the profile of the average speed along the 
road for both directions and have compared the right and the left lane. It is expected that in the left lane, the speed difference is larger (it is closer to the incident) and the acceleration is higher, because in the left lane are no trucks (which accelerate slower) and the left lane is probably occupied by the more aggressive drivers. We performed this analysis for the two locations where two lanes are available: Apeldoorn westbound and Gorinchem eastbound.

\subsubsection{Queue discharge rate}

The headways give an indication of the reciprocal value of the queue discharge rate. However, in determining the headways we only consider the vehicles that could be tracked over the whole stretch. To obtain a more reliable estimate of the queue discharge rate and the fluctuations thereof, for all passing vehicles the passing time is recorded. This is done using the video data; also the counts of the loop detectors are used, but they do not distinguish between cars and trucks. From these passing times we computed flows by aggregating them over time and, if relevant, over the carriageway.

Queue discharge rate stochastic while the distribution depends on external factors (such as weather, road geometry) and individual characteristics of the drivers. Usually, it is characterized by the median value: the maximum queue discharge rate that is obtained in half of the cases. For one measurement site, the external conditions are fixed. The variability of the queue discharge rate indicates the extent of the inter-driver differences. Not all aggregation intervals are suitable to estimate the queue discharge rate. For instance, there should be a queue of cars waiting to pass and the flow should be uninterrupted which is not always the case at the incident location near Gorinchem. If there is no constant queue discharge in some aggregation interval, this interval should be ignored in estimating the queue discharge rate. Therefore, the amount of data that should be ignored increases with the aggregation time. For this reason, we have taken a relatively short aggregation time of 30 seconds. Using shorter intervals increases the spread of the measurements, but will not change the median value.

Using 30 seconds intervals, there are 35-40 aggregation intervals for the location near Apeldoorn (the number of usable intervals depends on the lane and direction) and 45 for the location near Gorinchem. To obtain a single value for the flow, all passing vehicles have been converted to passenger car equivalents. Hence, the passing of a truck is counted as 1.5 passenger car according to the Highway Capacity Manual (2000). The flows have been converted in this way to passenger car units per hour lane, pcu/lane-h.

Only locations with an bottleneck are useful for determining the outflow capacity. These are:

- Apeldoorn eastbound - carriageway

- Apeldoorn westbound - right lane 
- Apeldoorn westbound - left lane

- Apeldoorn westbound - carriageway

- Gorinchem westbound - left lane

The first value is for the amount of traffic that passes the incident near Apeldoorn, the eastbound direction. The capacity is a result of the driver's behaviour. Adding the flows of the shoulder and the right lane (used intermittently) gives information of the realized queue outflow discharge rate if these two lanes are available. This way, we have not obtained a capacity value for each lane. However, the value we have got for the carriageway is a result of drivers' behaviour to pass the incident location. The resulting flow values have been divided by 2 to get an outflow capacity per lane.

Traffic in the non-incident direction used both lanes continuously. Therefore, we could compute a capacity value for each of the 2 lanes, as well as the average outflow capacity (distribution) per lane. The fifth capacity value is the capacity of the remaining lane passing the incident at the incident location near Gorinchem.

Let us finally remark that the queue discharge rate is lower than free flow capacity, the maximum number of passenger car equivalents per unit of time that can pass a cross-section of a road in free flow, which is usually obtained before congestion sets in. This phenomenon is called the capacity drop. It is described extensively in literature and estimations for the reduction vary, but are typically around 10\% (Cassidy and Bertini, 1999, Chung et al., 2007, Dijker et al., 1997, Hall and Agyemang-Duah, 1991). In the analyses we will only use the queue discharge rate.

\section{Results}

The data have been analysed in the way described in the last section. This section presents the results and is divided into two subsections. The first one presents the results on the level of individual cars, the microscopic flow characteristics, and the second one presents the findings on an aggregated level, the macroscopic flow characteristics.

\subsection{Microscopic properties}

Let us first consider the microscopic characteristics determined from the helicopter data. 


\subsubsection{Headways}

Analysing the flows at the level of detail of individual vehicles, we could obtain the headway distribution on a cross-section. As an example, consider the distribution of the 123 headways on a cross-section at left lane of Apeldoorn westbound, shown in Fig. 4. Both the cumulative curve and the histogram are shown, which respectively show what part of the headways is lower than a certain value, and the fraction of vehicles having a headway according to the bin size. The majority has a headway of around 2 seconds, derived from the highest bar, while another part chooses a headway at around 4 seconds. Similar distributions are found for the other locations; the data of all locations are summarized in Table 4.

Table 4. The median headways and standard deviation

\begin{tabular}{llll}
\hline & \multicolumn{2}{l}{ Apeldoorn westbound Apeldoorn westbound } & Gorinchem westbound \\
\hline Lane & Right & Left & Left \\
Median headway & $3.2 \mathrm{~s}$ & $2.1 \mathrm{~s}$ & $3.7 \mathrm{~s}$ \\
Standard dev. & $2.7 \mathrm{~s}$ & $0.9 \mathrm{~s}$ & $2.4 \mathrm{~s}$ \\
Normal & $1.9 \mathrm{~s}$ & $1.9 \mathrm{~s}$ & $2.0 \mathrm{~s}$ \\
\hline
\end{tabular}

Both sites are no bottlenecks in normal conditions, so an alternative method was used to derive the queue discharge rate and from that the minimum headways in a bottleneck. in An intensive research studying 2 years of data showed that the median queue discharge rate of Dutch motorways is around 1875 veh/lane-h (Heikoop et al., 2007) which implies a median queue discharge headway for normal traffic of 1.9 seconds in the Netherlands, which is used as reference for Apeldoorn site.

Also the Gorinchem is no bottleneck in normal conditions, but there are queues spilling back to the site. Therefore, both the congested branch and the free flow branch of the fundamental diagram could be fitted on measurements from loop detector data using the functions proposed by $\mathrm{Wu}$ (2002). The intersection of these two branches gives a queue discharge rate of 1960 veh/lane-h, which implies a median queue outflow headway of $2.0 \mathrm{~s}$.

The significance of the differences in a distribution can be shown using a Kolmogorov-Smirnov test. There is a significant difference if for any value of the variable the difference between the two cumulative distributions is larger than the Kolmogorov distance (Chakravarti et al., 1967). In this case, however, only one level of the cumulative distribution for the reference situation is known, namely that $50 \%$ of the headways is lower than at 1.9 respectively 2.0 seconds for Apeldoorn or Gorinchem. These points are a single point at each of the respective distribution functions. The distance of this point of the reference distribution function to the distribution function of the headways at the respective incident site is now computed. This distance is larger than the Kolmogorov distance for a significance level of $5 \%$, which means that the two distributions are significantly different. This test was repeated for all lanes at the incident sites, which showed that the 
headways at each site differ significantly from the headways under normal driving. Therefore, hypothesis 1 is accepted.

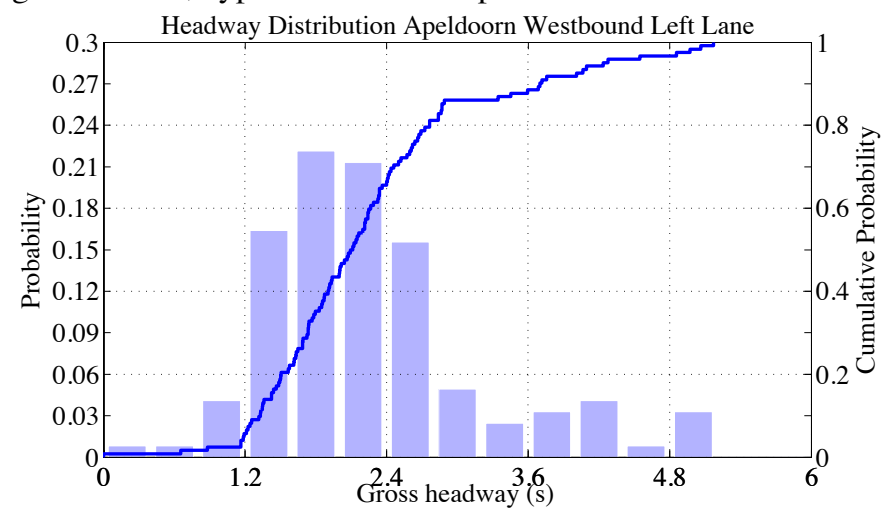

Fig. 4 The headway distribution for Apeldoorn westbound left lane.

\subsubsection{Reaction times}

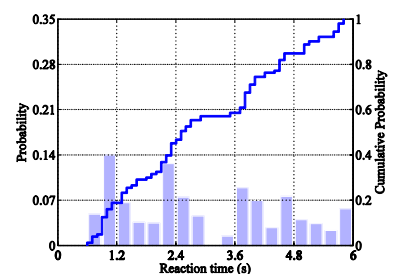

a

Fig. 5 The distribution of reaction times during an incident (a) and the normal conditions (b)

Fig. 7 shows the distribution of the fitted reaction times over the different drivers, in a graph similar to Fig. 4; the values are summarized in Table 5. Note that the reaction time $\tau_{r}$ should not be interpreted as the time one physically needs to process an input. $\tau_{r}$ can be interpreted as the time a driver takes before an input has effect on his driving operations. For the reference situation in Fig. $7 \mathrm{~b}$, reaction times, presented in Ossen et al. (2006), are shown without a weighting factor, but for the incident measurements an extra weight is used for fitted parameters which are more reliable according to equation (3). In fact, the line in Fig. 7a shows which part of the weight of the fitted reaction time estimates is lower than a value and the 
bars show how much weight of the reaction time estimates is located in each of the bins.

The reaction times are distributed similar to the headways. Similarly, there are two values around which the reaction times centres, 2 seconds and 5 seconds, as can be seen for instance for the left lane of Apeldoorn westbound (Fig 7a). A Kolmogorov-Smirnov test is performed on each of the incident reaction time distributions and it shows that these distributions all differ significantly at a significance level of 5\% from the reaction times at a non-incident location. In fact, the reaction time at the incident sites is time is much larger and therefore hypothesis 2 is accepted.

Table 5. The reaction times

\begin{tabular}{lllll}
\hline & Apeldoorn westbnd & \multicolumn{2}{l}{ Apeldoorn westbnd Gorinchem eastbnd } & Netherlands normal \\
\hline Lane & Right & Left & Right & Average \\
Mean & $3.9 \mathrm{~s}$ & $2.9 \mathrm{~s}$ & $3.8 \mathrm{~s}$ & $1.3 \mathrm{~s}$ \\
Std. dev. & $1.4 \mathrm{~s}$ & $1.6 \mathrm{~s}$ & $1.3 \mathrm{~s}$ & $1.0 \mathrm{~s}$ \\
\hline
\end{tabular}

\subsection{Macroscopic properties}

Let us now discuss the main macroscopic properties of the flow during the incident and compare it with normal circumstances.

\subsubsection{Average Speed}

The speed profile for Apeldoorn and Gorinchem is shown in Fig. 8. The bold line is the median speed (of the cars) for all positions along the road. It also shows the interval bounds (60\% of the speeds lies within the gray area).

At the Apeldoorn site the average speed drops when drivers approach the incident site. After people have passed a certain point, they start accelerating again. This point appears to be the point providing the best view on the incident location. At this point, the average speed in the right lane is actually higher than in the left lane; in fact, the minimum speeds are $7.8 \mathrm{~m} / \mathrm{s}$ for the right lane and $6.2 \mathrm{~m} / \mathrm{s}$ in the left lane. However, the vehicles in the left lane accelerate faster and they have a speed of $18.9 \mathrm{~m} / \mathrm{s}$ when they reach the point at which they drive out of the picture, whereas the average speed in right lane is just $14.6 \mathrm{~m} / \mathrm{s}$ at this point. In order to test the difference in the speed reduction in the right and left lane, we used $t$-test, which yields a $P$-value of $5^{*} 10^{-6}$ indicating a significant difference.

In Gorinchem dataset there is no queue in the non-incident, eastbound direction. The speeds (Fig. 8b) show that people nevertheless reduce their speed. Drivers have passed the most eastbound wreck at 220 meters. The minimum of the 
average speed lies at a point a little downstream, at around 250 meters. This is possibly caused by looking at the traffic jam (including the merging) at the other side. In addition, there is less incentive to accelerate quickly. Namely, contrary to the Apeldoorn site, the drives do not drive in congestion. Therefore, they might not have realised for a second that their speed has reduced.

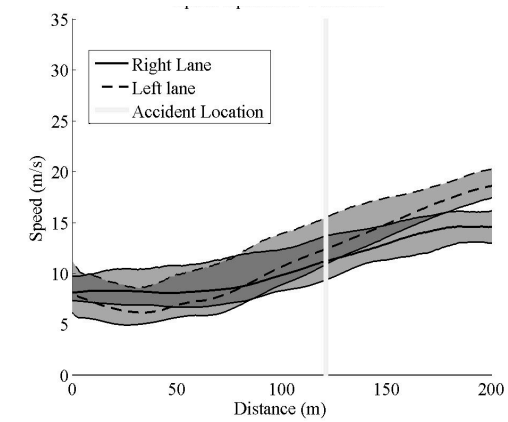

a

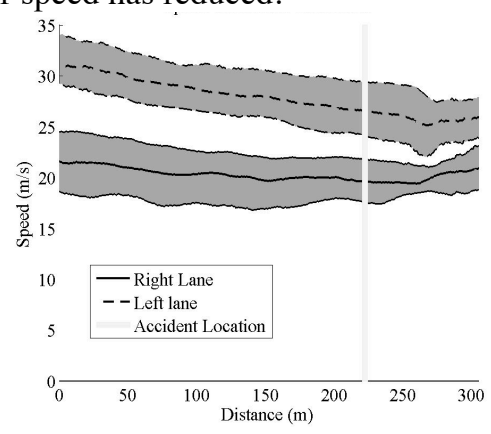

B

Fig. 6 The speed profile along the road for Apeldoorn westbound and Gorinchem eastbound as a function of the distance along the road

Only the start of the acceleration lies within the area that is captured on video. At this location, we therefore focus on the speed reduction. The average speed in the left lane drops from $31.1 \mathrm{~m} / \mathrm{s}$ to $24.8 \mathrm{~m} / \mathrm{s}$, which is a difference of $6.3 \mathrm{~m} / \mathrm{s}$. In contrast, the average speed in the right lane reduces by just $2.6 \mathrm{~m} / \mathrm{s}$, from $22.0 \mathrm{~m} / \mathrm{s}$ to $19.4 \mathrm{~m} / \mathrm{s}$. A $t$-test has shown that this speed reduction is significantly larger in the left lane than in the right lane with a $P$-value of $1 * 10^{-4}$. This difference in free speed can partially be explained by the European law which requires the drivers, in non-congested conditions, to keep the right lane when possible, and to overtake at the left lane. Therefore, since the traffic is still in free flow conditions, the right lane has a lower speed than the left lane. Additionally, 25\% of the vehicles in the right lane are trucks with a lower speed limit, whereas there are no trucks in the left lane. To confirm the idea that trucks influence the magnitude of the speed reduction, we analysed the difference in speed between trucks and passenger cars in the right lane, we have found that trucks keep a more constant speed. Passenger cars enter the area at higher speed than the truck and decelerate to a lower speed than the trucks.

So, at the location of the incident, the average speed of the vehicles drops, which confirms hypothesis 3 . Moreover, average speed in the left lane drops more than the average speed in the right lane which can be explained in three ways. Firstly, they are probably more distracted since they are closer to the incident. Secondly, there could be a psychological difference between drivers in the right and the left lane. More aggressive drivers are more likely to drive in the left lane. Thirdly, the less powerful cars and trucks, having a slower acceleration, probably stay in the right lane. 


\subsubsection{Queue discharge rates}

Fig. 9 is a boxplot of the queue discharge flows. The middle line indicates the median flow value; the box is made of horizontal lines at the $25 \%$ and $75 \%$ percentile. The whiskers (at the outside) give the total range of the values. In addition, the queue discharge rates of the incident locations are stated in Table 6. As normal queue discharge rates we use the values as found in section 5.1.1, namely 1875 $\mathrm{veh} / \mathrm{h}$-lane for the Apeldoorn site and $1760 \mathrm{veh} / \mathrm{h}$-lane for the Gorinchem site.

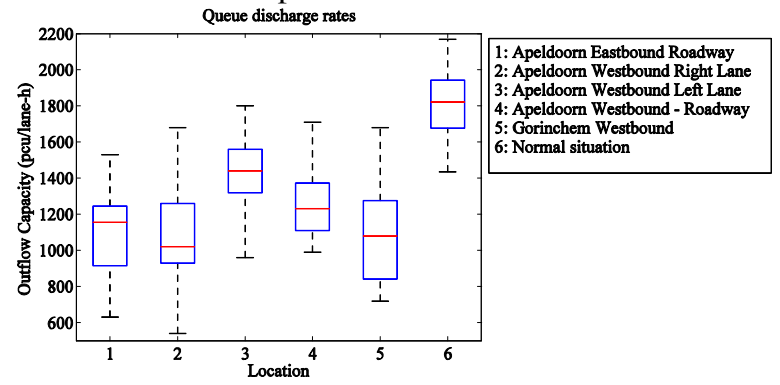

Fig. 7 The queue discharge rates for the different locations.

As expected, the queue discharge flow values for the locations with an incident drop to values under the usual ones based on the remaining lanes (Table 6). The reference queue discharge rates are in vehicles/lane-h, rather than pcu/lane-h. To be sure that the incident values are not marked significantly different wrongly, we need to be sure not to overestimate the truck share in the reference values, so we have conservatively processed the value as was the truck share $0 \%$, which means that, by converting the values to pcu-values, the reference estimate is certainly not too high. Z-tests have shown that for each of the 5 locations the queue discharge rate is significantly different (5\% significance), from the normal queue discharge rates. The stated expected capacity is the reference free flow capacity for the number of lanes that is open. So, for example, in the case of Gorinchem, the Table 6 states the reference capacity of one lane. Thus, the reduction of capacity cannot be explained by the reduction of number of lanes. Since we compared outflow values with the outflow capacities, it is also not the capacity drop. Thus, hypothesis 4 could be accepted.

In the first case, Apeldoorn eastbound, one of the two remaining lanes is the shoulder lane. This has two consequences for the estimation of the capacity reduction. First, the reference capacity is probably lower than the capacity for two fullwidth lanes. Secondly, as described in section 3.2, the use of both lanes at this location varies in time: in some periods the shoulder lane is used, in some periods 
the right lane is used and in some periods, both of them are used. If they were using both lanes continuously, the flow would probably have been higher. However, the data represents the drivers' behaviour around the incident, including their lane choice.

This reduction may be caused by "rubbernecking", the fact that people are distracted by watching what has happened. Another possibility is that people drive extra carefully since there are people working at the roadway. Compared to the normal queue discharge rate, also stated in Table 6 , the queue discharge rate reduces by $25-40 \%$. Other publications as by Qin and Smith (2001), or the Highway Capacity Manual show lower reductions, even when compared to the higher free flow capacity. The confidence bounds for the values in these references are unclear, and therefore it is impossible to tell whether the difference is significant. A possible explanation for a larger reduction found in this study is the high queue discharge rate in normal conditions in the Netherlands. A similar congestion flow would then lead to a higher reduction.

It is remarkable that the resulting maximum flow rates at the incident near Apeldoorn are about the same for the eastbound and the westbound direction, whereas in the eastbound direction one lane is blocked. Drivers will use the shoulder lane from time to time. Blocking of lanes and looking at the incident causes the same reduction of flow at the carriageway with the incident as the rubbernecking on the carriageway for the opposite direction.

Table 6 Queue discharge rates for different locations

\begin{tabular}{|c|c|c|c|c|c|}
\hline Location & Lanes & $\begin{array}{l}\text { Median } \\
\text { pcu/lane-h }\end{array}$ & $\begin{array}{l}\text { St dev } \\
\text { pcu/lane-h }\end{array}$ & $\begin{array}{l}\text { Expected } \\
\text { without } \\
\text { rubbernecking } \\
\text { pcu/lane-h }\end{array}$ & $\begin{array}{l}\text { Percent of } \\
\text { queue dis- } \\
\text { charge rate } \\
\text { remaining }\end{array}$ \\
\hline Apeldoorn eastbound carriageway & 2 & 1170 & 239 & 1875 & $59 \%$ \\
\hline Apeldoorn westbound right lane & 1 & 1020 & 249 & 1875 & $58 \%$ \\
\hline Apeldoorn westbound left lane & 1 & 1440 & 195 & 1875 & $76 \%$ \\
\hline Apeldoorn westbound carriageway & 2 & 1230 & 163 & 1875 & $66 \%$ \\
\hline Gorinchem westbound & 1 & 1080 & 326 & 1760 & $61 \%$ \\
\hline
\end{tabular}

\section{Conclusions and Discussion}

This contribution proposes a new conceptual model for driving in near an incident. The model states that a shift of the driver's attention leads to a different traffic characteristics. To prove the model, microscopic data of traffic operations at two incident sites were was used. We accepted the following hypotheses: 
1. The distribution of headways in a bottleneck caused by an incident is different compared to a bottleneck in normal traffic and the mean headway is larger.

2. The reaction time distribution of drivers is different compared to normal traffic and the mean is larger

3. The sight of an incident site reduces the average speed.

4. The outflow capacity at the incident site is lower than the outflow capacity under normal conditions for the same roadway geometry.

This supports the theory that drivers shift their attention towards the incident when passing by the incident site. We found evidence implying that that there may be two groups of drivers, those who have a slightly reduced attention level and a smaller group with a considerably reduced attention level, shown by a large headway and reaction time.

These behavioural changes lead to a reduction of the queue discharge rate. At the incident site, the queue discharge rate per lane is $60-75 \%$ of normal queue discharge rate per lane. This holds for both directions: the incident direction and the incident direction, implying that when a lane is blocked, the queue discharge rate of the carriageway reduces by more than $25 \%$.

For practical applications a simulation model for traffic flows around incidents would be useful. Every simulation model, both microscopic and macroscopic, needs calibration and validation. It is difficult to calibrate a simulation model for an incident situation, since there are few incident situations, which all are different. The findings presented here are based on observations of many drivers. Drivers at another incident might drive differently.

Future research could investigate the psychological effects in a more controlled situation, as for instance a driving simulator. Other future work includes the lane choice behaviour around accidents.

Acknowledgments This research was supported by the research program Next Generation Infrastructures, the Transport Research Centre Delft and the research program Tracing Congestion Dynamics - with Innovative Data to a Better Theory (sponsored by the Dutch Foundation of Scientific Research MaGW-NWO). The comments of the anonymous reviewers were also gratefully acknowledged.

\section{References}

Highway Capacity Manual (2000). Washington D.C., Transportation Research Board.

Cassidy, M. J. \& Bertini, R. L. (1999) Some traffic features at freeway bottlenecks. Transportation Research Part B: Methodological, Vol. 33, no. 1, pp. 25-42.

Chakravarti, I. M., Laha, R. G. \& Roy, J. (1967) Handbook of methods of applied statistics: techniques of computation, descriptive methods, and statistical inference. , New York, John Wiley and Sons. 
Chung, K., Rudjanakanoknad, J. \& Cassidy, M. J. (2007) Relation between traffic density and capacity drop at three freeway bottlenecks. Transportation Research Part B: Methodological, Vol. 41, no. 1, pp. 82-95.

Dijker, T., Bovy, P. H. L. \& Vermijs, R. G. M. M. (1997) Car-following under non-congested and congested conditions. Delft, Delft University of Technology.

Hall, F. L. \& Agyemang-Duah, K. (1991) Freeway Capacity Drop and the Definition of Capacity. Transportation Research Record: Journal of the Transportation Research Board No.1320, pp. 9198.

Heikoop, H., Hoogendoorn, S. P. \& Martens, G. J. (2007) Onderzoek Verkeersafwikkeling en capaciteitswaarden discontinuïteiten Autosnelwegen. Rijkswaterstaat, Adviesdienst Verkeer en Vervoer.

Hoogendoorn, S., Van Zuylen, H. J., Schreuder, M., Gorte, B. G. H. \& Vosselman, M. (2003) Microscopic traffic data collection by remote sensing. Transportation Research Record: Journal of the Transportation Research Board No.1885, pp. 121 - 128.

Hoogendoorn, S. P. \& Van Lint, J. W. C. (2007) Estimation of Car-Following Models using Prior Information. In Proceedings of the IEEE conference on Intelligent Transport Systems.

Knoop, V. L., Hoogendoorn, S. P. \& Van Zuylen, H. J. (2008) Capacity Reduction at Incidents: Empirical Data Collected from a Helicopter. Transportation Research Records. Accepted for publication.

Kwon, J., Mauch, M. \& Varaiya, P. (2006) The components of congestion: delay from incidents, special events, lane closures, weather, potential ramp metering gain, and excess demand. In Proceedings of the 85th annual meeting of the Transportation Research Board, Washington.

Ossen, S., Hoogendoorn, S. \& Gorte, B. G. H. (2006) Interdriver Differences in Car-Following: A Vehicle Trajectory-Based Study. Transportation Research Record: Journal of the Transportation Research Board No.1965, pp. 121-129.

Ossen, S. J. L. (2008) Longitudinal Driving Behavior: Theory and Empirics, TRAIL Thesis Series PhD thesis, Delft, Delft University of Technology.

Qin, L. \& Smith, B. L. (2001) Characterization of accident Capacity Reduction. University of Virginia.

Schaap, N., Van Arem, B. \& Van Der Horst, R. (2008) Drivers' Behavioural Reactions to Unexpected Events. In Proceedings of 10th TRAIL Congress - TRAIL in Perspective, Rotterdam, the Netherlands.

Sinha, P., Mohammed Hadi, P. E. \& Amy Wang, E. I. (2007) Modeling Reductions in Freeway Capacity due to Incidents in Microscopic Simulation Models. In Proceedings of 86th Annual Meeting of the Transportation Research Board, Washington D.C.

Tampère, C. M. J. (2004) Human-kinetic multiclass traffic flow theory and modelling, PhD Thesis thesis, Delft University of Technology.

Thiemann, C., Kesting, A. \& Treiber, M. (2008) Estimating Acceleration and Lane-Changing Dynamics Based on NGSIM Trajectory Data. In Proceedings of the 87th Annual Meeting of the Transportation Research Board, Washington D.C.

Toledo, T., Koutsopoulos, H. N. \& Ahmed, K. I. (2007) Estimation of Vehicle Trajectories with Locally Weighted Regression. In Proceedings of the 86th Annual Meeting of the Transportation Research Board, Washington D.C.

Wu, N. (2002) A new approach for modeling of Fundamental Diagrams. Transportation Research Part A: Policy and Practice, Vol. 36, no. 10, pp. 867-884. 